\title{
Quick Report on On-Board Demonstration Experiment for Autonomous-Visual-Guidance Camera System for Space Debris Removal
}

\author{
By Shinichi KImURA, ${ }^{1)}$ Yuta HoriKawA, ${ }^{2)}$ and Yasuhiro KataYAmA ${ }^{2)}$ \\ ${ }^{1)}$ Department of Electrical Engineering, Tokyo University of Science, Chiba, Japan \\ 2) JAXA, Tsukuba, Japan
}

(Received June 22nd, 2017)

\begin{abstract}
Recently, problems involving space debris have become more serious. According to NASA research, the volume of space debris is projected to increase, even if no new satellites are launched. Therefore, debris-removal satellites must be developed immediately. A mandatory function of a debris-removal satellite is to recognize and approach target debris. Thus, visual guidance using image processing is being considered as an effective means of guiding debris-removal satellites toward unresponsive targets. A small satellite is suitable for use as a debris-removal satellite; however, because of weight and/or size limitations, the installation of certain cameras in small satellites is difficult. Thus, we have developed a compact camera system that can perform on-board image processing, by expanding the functionality of an existing camera system to enable it to acquire the multi-direction images required during the satellite-debris rendezvous process. Experiments were conducted using our proposed system on the H-2 Transfer Vehicle (HTV) as part of an electrodynamic-tether experiment. This paper presents a brief report on the results of this HTV flight experiment.
\end{abstract}

Key Words: Space Debris, Mitigation, Visual Guidance, International Space Station

\section{Introduction}

In recent years, problems associated with space debris have become more serious, as space development has become more active. Furthermore, because collisions among existing debris generate new items of debris, the volume of space debris is projected to exponentially increase, even if no new satellites are launched. ${ }^{1)}$ For example, in 2009, the Iridium 33, collided with a Russian satellite, Kosmos-2251. The collision generated more than 2,000 pieces of traceable debris. If these fragments collide with the International Space Station (ISS) or other satellites, serious problems will arise. In fact, impact damage has already been observed on the ISS. ${ }^{2)}$ Thus, management of spacecraft activity in orbit is difficult without debris surveillance. Further, according to research conducted using the NASA long-term debris evolutionary model, LEGEND, it is necessary to remove five large-size items of debris per year to maintain the current space environment. ${ }^{3)}$ Therefore, satellites for debris removal must be developed immediately.

An imperative function of a debris-removal satellite is to autonomously recognize and approach the target debris by sensing its distance and movement. The debris-removal satellite cannot be expected to communicate with the debris, and, owing to additional communication limitations, real-time remote operation of the debris-removal satellite from ground stations is impractical. Thus, visual guidance using image processing is being considered as an effective method of guiding debris-removal satellites toward unresponsive targets. For example, image processing can be used to estimate the distance between a debris-removal satellite and items of debris, or to estimate the debris movement.

A small satellite is suitable for use as a debris-removal satellite, because it can be realized at a very low cost and within a short period. In addition, small satellites are often equipped with a camera for situation monitoring, and such satellites have many launch opportunities. However, because of weight or size limitations, the installation of certain cameras in small satellites is difficult.

To overcome these difficulties, we have developed a relatively compact camera system that can perform on-board image processing. To develop this system within a short period, we decided to expand the Interplanetary Kite-craft Accelerated by Radiation of the Sun (IKAROS) camera system for use as the visual guidance system of a debris-removal satellite. The camera system in question was deployed on the small solarelectric power-sail demonstrator IKAROS, which was launched in 2010. The IKAROS camera system can acquire high-resolution digital images, having a high calculation capability and being powered by a PowerPC processor and a Linux Operating System (OS). ${ }^{4-6)}$ Visual guidance software was developed and implemented as a Linux application on the IKAROS camera system.

In debris-removal operations, the rendezvous process has two phases: the debris-removal approach phase, which requires high-resolution images to acquire detailed information about the debris, and the homing phase, which requires wide-area images in order to locate debris over a large area. Thus, we expanded the IKAROS camera system to facilitate acquisition of the multi-direction images required during a satellite-debris rendezvous process. The resultant camera system has two image lens systems, i.e., the High-Resolution (HRCAM) and Wide-Angle (WACAM) camera systems. Further, it possesses an IF board that controls the two image lens systems using a processing system.

We planned to demonstrate the performance of our proposed system on the H-2 Transfer Vehicle (HTV) as part of an 
ElectroDynamic-Tether (EDT) experiment. ${ }^{7-9)}$ The developed camera system can perform two types of real-time image processing, using HRCAM or WACAM. In the context of the HTV mission, estimation of the tether-endmass distance during tether-endmass ejection was planned using HRCAM; this corresponds to the debris-removal approach phase. A second operation involved tether motion recognition to detect a marker attached to the tether; this operation required the use of WACAM and corresponded to the target recognition and orbit estimation processes during the homing phase of a debrisremoval mission. As an advanced mission, we also planned to acquire images of the ISS using this camera, when the HTV rendezvoused with the ISS.

This paper presents brief reports on the results of the flight experiment for the autonomous-visual-guidance camera system for space-debris removal conducted on the HTV.

\section{Kounotori Integrated Tether Experiment (KITE)}

The Japan Aerospace Exploration Agency (JAXA) conducts research and development on debris-removal satellites. ${ }^{10)} \mathrm{A}$ deorbit technique using an EDT is necessary, as well as a key technology, for debris-removal systems. Thus, the Kounotori Integrated Tether Experiment (KITE) has been designed to demonstrate the performance of the current EDT design.

In the KITE mission, a component called "Endmass" that is attached at the end of the electric dynamic tether is ejected from the HTV, and the electric dynamic tether is deployed by the motion of the "Endmass". Therefore, the motion parameters of the "Endmass," such as ejection direction and speed, are especially important to analyze the deploying process of the electric dynamic tether. We decided to acquire this information by using images captured by the camera that is mounted on the surface of the HTV. Therefore, the first mission of the KITECam is to acquire the motion information of the "Endmass".

Because the electric dynamic tether is very long, the motion of the "Endmass" cannot be acquired by the images captured by the KITE-Cam after the electric dynamic tether is deployed. Therefore, we attached three small markers on another end of the electric dynamic tether. The tether motion can be analyzed using the images of these markers after the electric dynamic tether is fully deployed.

The Kounotori or HTV spacecraft can communicate with a ground station, can supply power, and is always visible. In addition, its launch schedules are guaranteed, and the use of components such as a rendezvous sensor simplifies the experimental system. These advantages enabled us to perform the planned experiment on the developed camera system using the HTV. Two main missions were planned: acquisition of tether-stretching data and drive current supply.

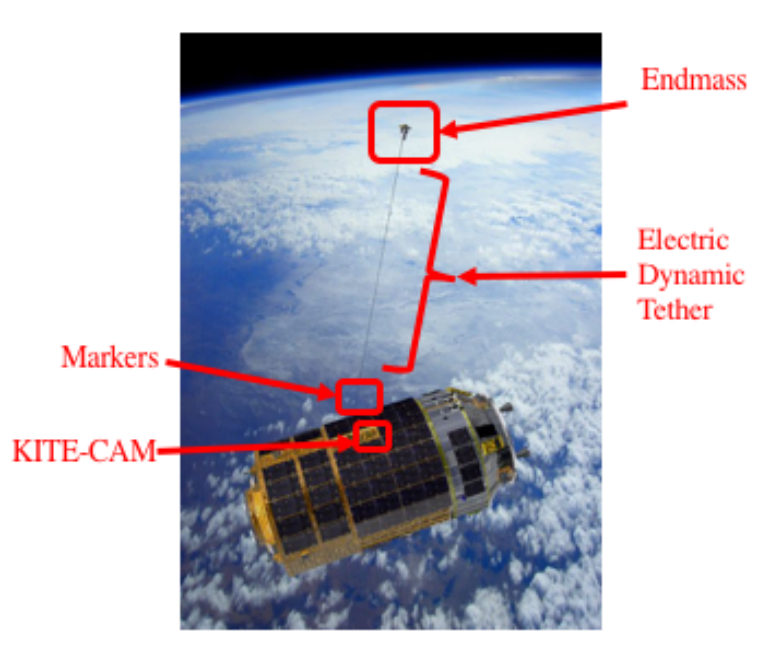

Fig. 1. Image of KITE mission (HTV with EDT) .

\section{Visual Guidance and Navigation System for Space Debris Removal}

\subsection{Structure}

The existing IKAROS camera system was expanded to facilitate acquisition of the multi-direction images required during a satellite-debris rendezvous process. As noted above, the two phases of the rendezvous process, i.e., the debrisremoval approach phase and the homing phase, require highresolution images to yield detailed information on the debris and wide-area images to locate debris over a large area, respectively. As a result of applicable weight or size limitations, however, installation of certain cameras in small satellites is difficult. Thus, for compactness, the camera system should employ a single processing system that controls the two image lens systems (HRCAM and WACAM). Use of a single processing system simplifies the electrical interfaces of the data handling unit, the telemetry and command interface, and the power conditioner unit. To control both of these image lens systems via the processing system, the developed camera system utilizes an IF board. Fig. 2 shows the KITE camera (hereafter referred to as "KITE-CAM"), which is an expansion of the IKAROS camera system design; the system specifications are listed in Table 1.

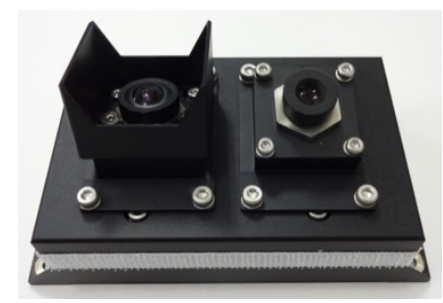

Fig. 2. KITE-CAM with HRCAM (left) and WACAM (right) lens systems.

Table 1. KITE-CAM specifications.

\begin{tabular}{ll}
\hline Size (excluding optics) & $124 \times 66 \times 32\left[\mathrm{~mm}^{3}\right]$ \\
\hline Mass & $0.35[\mathrm{~kg}]$ \\
\hline
\end{tabular}


KITE-CAM missions can differ widely in terms of target, distance, or direction. Thus, we designed and developed image lens systems that can be employed in each scenario through application of image processing techniques.

For example, during tether-endmass ejection, the endmass is first imaged at distances of up to $100 \mathrm{~m}$ from the HTV. Highresolution images are required to allow estimation of the tetherendmass distance from the endmass area. Therefore, the HRCAM image lens system is equipped with a telephoto lens with an 8-mm focal length, and its optical axis is inclined by $+10^{\circ}$.

Next, markers within $2 \mathrm{~m}$ from the base of the tether are imaged. Wide-area images are required to allow the tether to swing by $\pm 60^{\circ}$ in the orbit plane. Therefore, the WACAM image lens system is equipped with a wide-angle lens with a 2$\mathrm{mm}$ focal length, and its optical axis is inclined by $-15^{\circ}$.

Table 2. KITE-CAM optics specifications.

\begin{tabular}{|c|c|c|}
\hline & HRCAM & WACAM \\
\hline Focal length & $8[\mathrm{~mm}]$ & $2[\mathrm{~mm}]$ \\
\hline Row resolution & \multicolumn{2}{|c|}{$1280[$ pixel] } \\
\hline Column resolution & \multicolumn{2}{|c|}{1024 [pixel] } \\
\hline $\begin{array}{l}\text { Vertical field-of-vision } \\
\text { (FOV) }\end{array}$ & $37.087\left[^{\circ}\right]$ & $106.606\left[^{\circ}\right]$ \\
\hline
\end{tabular}

\subsection{Electrical system}

The developed camera system has the two image lens systems described above, and employs an IF board to control these systems using a processing system. The wiring is reduced compared to the IKAROS design through use of a serializer and deserializer, and alternates between the two image lens systems via a bus switch. Fig. 3 is a system diagram.

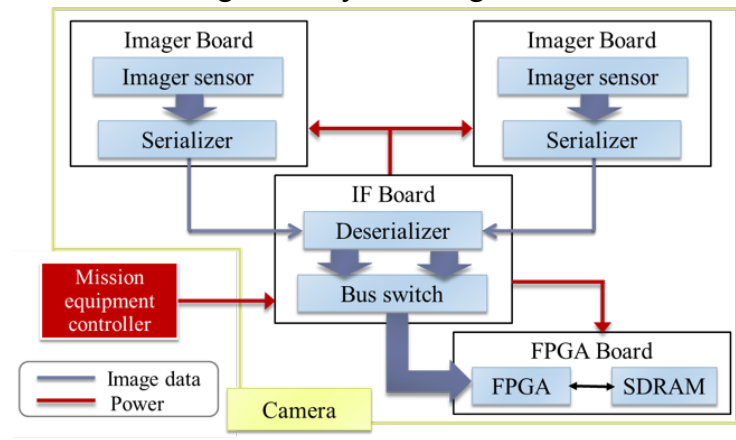

Fig. 3. System diagram. FPGA: Field-programmable gate array; SDRAM: Synchronous dynamic random access memory.

The two image lens systems contain the same type of imager board. The image sensor (OV9630, OmniVision) is identical to that used in IKAROS. The IF board is equipped with deserializers and bus switches.

Fig. 4 shows the field-programmable gate array (FPGA) board. A Xilinx FPGA Virtex-2 Pro is employed as the main camera processor. The reliability of this FPGA has been proven on the IKAROS mission. The logic synthesis file of the FPGA is based on the IKAROS camera and is modified to suit the KITE-CAM IF board. This FPGA board can connect to the IF board in a $5-\mathrm{mm}$ stack.

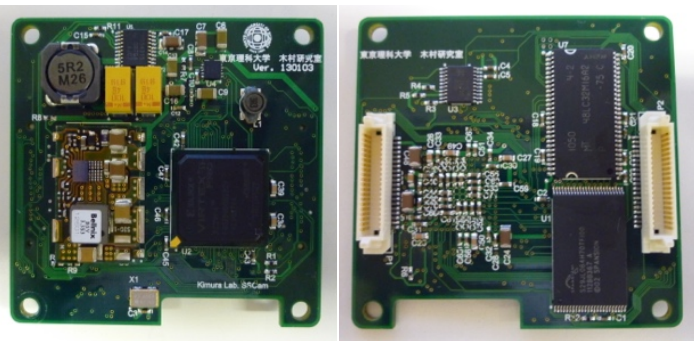

Fig. 4. FPGA board.

\subsection{Image processing}

KITE-CAM missions require near-real-time downlink data. However, because of bandwidth limitations, downlinking of unprocessed image data is difficult. Therefore, the data volume is reduced using image processing. Then, the acquired images are saved in the negative-AND (NAND) flash memory. The data in the memory are downlinked after the experiments and analyzed on the ground.

Two types of real-time image processing are available. The first estimates the tether-endmass distance during tetherendmass ejection, which corresponds to the debris-removal approach phase. The HRCAM is used for this mission. The acquired data are the area of the tether-endmass and its corner. Note that the tether-endmass area in the acquired image will be reduced if the tether-endmass moves away from the HTV. Thus, imaging at high frequencies and image saving are very important, because the area variation is large.

The other type of real-time image processing is tether motion recognition to detect a marker attached to the tether, which corresponds to the target recognition and orbit estimation processes during the homing phase. The WACAM is used for this mission. The acquired data are the marker areas and positions in the image. This process uses a smoothing filter, binary image processing, and label processing. The data representing the two labeled areas of the largest area are downlinked. Note that, during this mission, the HTV position in its orbit around the Earth causes considerable variation in the lighting conditions.

\subsection{Development environment}

The Linux (Kernel 2.4) OS is employed in the image processor. Linux is very flexible and various free open-source applications can be utilized effectively with this OS, which can shorten the development period. In addition, use of a memory management unit and Linux, which are installed in the central processing unit (CPU), can ensure powerful memory protection and prevent important data from sudden destruction.

\section{Results}

\subsection{Operation results}

To test the target-recognition image processing application, images obtained via a JAXA imaging experiment, which was performed with a marker developed by JAXA and under different illumination conditions, were examined. Two target markers were considered, and we wished to obtain information about the area and aspect ratio of each marker. The aspect ratio 
was calculated using the formula:

$$
\frac{\mid \text { height }- \text { width } \mid}{\max \{\text { height, width }\}} \text {. }
$$

This formula indicates that the nearer the value to 1 , the more circular the form. In this application, an object was regarded as a marker if the aspect ratio was less than 7 . By comparing the output results with the images, we confirmed that increasing and decreasing areas could be accommodated by the proposed system and the aspect ratio could be obtained. Fig. 5 shows the coordinate system used in this experiment.

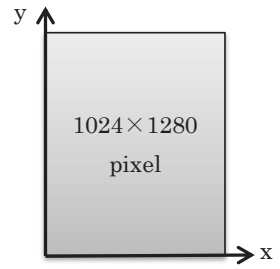

Fig. 5. Coordinate system used in target recognition application.

Table 3 lists the results of this test, and Figs. 6-8 show the images used for this operation. The table and figures indicate that information about the marker areas and aspect ratios were obtained. Note that the aspect ratio of Image $1\left(1 \times 10^{4}[1 \mathrm{x}]\right)$ was higher than those of the other images, because of the light beam. However, we are not concerned by this relatively high aspect ratio, because it remains less than 7 . Therefore, the required information can be obtained via this image processing application.

\begin{tabular}{lccc} 
& Table 3. & Operation results. & \\
\hline & Image 1 & Image 2 & Image 3 \\
\hline Illuminance [1x] & $1 \times 10^{4}$ & $1 \times 10^{3}$ & $1 \times 10^{2}$ \\
\hline Area size [pixels] & 422 & 151 & 64 \\
\hline Aspect ratio & 5 & 1 & 2 \\
\hline
\end{tabular}

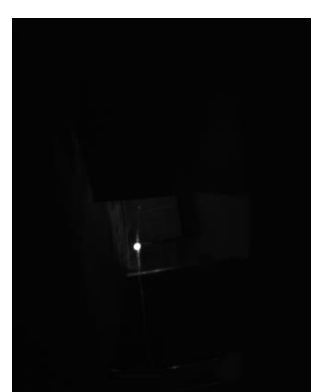

Fig. 6. Image 1 CJAXA (illuminance: $1 \times 10^{4} \mathrm{~lx}$ ).

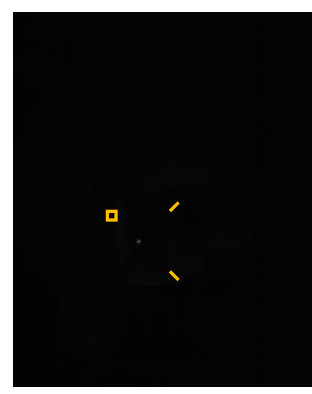

Fig. 8. Image 3 CJAXA. (illuminance: $1 \times 10^{2} \mathrm{~lx}$ )

\subsection{Experimental results in brief}

KITE-CAM was launched together with other KITE equipment on Dec 6th, 2016, as the 6th HTV flight. The KITE mission performed a retuning flight from Jan **th to **th, 2017 Unfortunately, the endmass failed to deploy, and the main image acquisition and analysis experiments of the KITE mission could not be performed on-board

However, as the HTV approached the ISS, KITE-CAM was activated and successfully acquired many images of the ISS as the advanced mission. Here, we present a small number of sample images obtained in this approaching phase (Figs. 10 and 11).

Firstly, we successfully confirmed periodical image acquisition by KITE-CAM. It is apparent that the ISS images were successfully acquired on an intermittent basis. Further, the ISS rendezvous process can be interpreted from these images (Fig. 10).
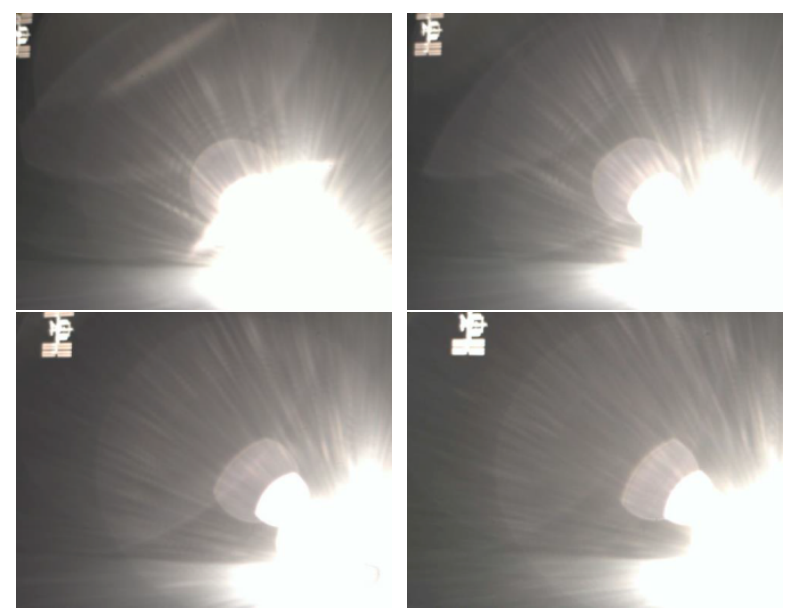

Fig. 10. Sample images acquired during ISS rendezvous process.

Unfortunately, because the image acquisition conditions, such as the KITE-CAM luminescence, were tuned for the KITE main missions involving acquisition of small-sized markers and endmasses, the sensitivity was too high to image the large ISS. Note that the ISS reflection rate is quite high and, thus, many of the acquired images tend to exhibit excessive luminescence, which is dependent on changes in the light orientation (Fig. 11). Thus, one of the most important points learned from these experiments is the importance of luminescence control, especially under high luminescence conditions. In our prior work on the REXJ project, we found that the on-board luminescence condition is significantly higher than that which can be automatically controlled by a commercial off-the-shelf (COTS) imager. However, the exposure- 0 mode can be utilized for such high-luminescence conditions. Unfortunately, this image acquisition mode was not installed on KITE-CAM. Therefore, we plan to further investigate methods to autonomously adapt those conditions. 


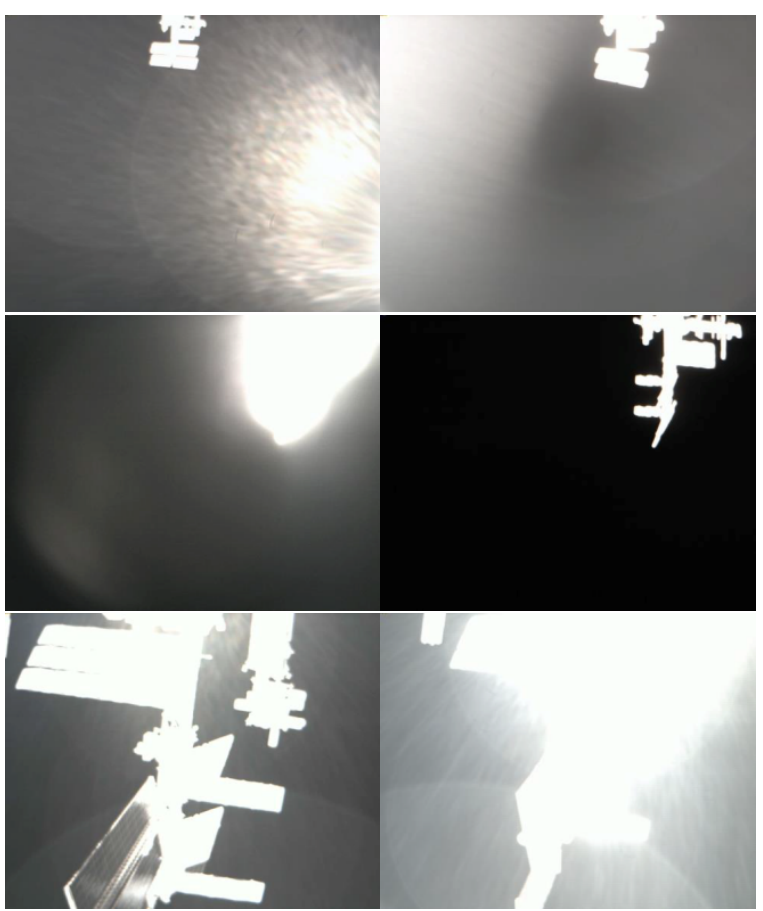

Fig. 11. Sample images acquired under normal- and high- luminescence conditions during ISS rendezvous process.

In addition, the KITE-CAM itself had difficulty switching from HRCAM to WACAM and was, thus, unable to acquire WACAM images. From analysis of the telemetry status, it is thought that the imager was functioning normally, because this device responded to the image acquisition condition command correctly. However, it is believed that the serializer may have caused the operation problem, based on on-ground simulation experiments. These findings suggest that the serializer treatment and harness require improvement for future applications.

\section{Conclusion}

In this paper, an outline of a developed visual guidance and navigation system for space debris removal is presented, along with a brief report on its demonstration mission, conducted on the HTV. For that mission, the developed system was referred to as "KITE-CAM." Unfortunately, we could not demonstrate the developed image processing technology in practical application because the endmass failed to deploy; however, we successfully acquired important data during the ISS rendezvous process. In future research, we plan to analyze these data and refine the devised visual guidance and navigation system for space debris removal for future applications.

\section{References}

1) Yasaka, T.: Space Debris Problem, Shokabo, Tokyo, 1997 (in Japanese).

2) Christiansen, E. L., Lear, D. M., and Hyde, J. L.: Recent Impact Damage Observed on ISS, the NASA Orbital Debris Program Office, Quarterly News, Houston, October 2014, 18(2014), Issue 4, pp.3-4.

3) Liou, J.-C.: An Update on LEO Environment Remediation with Active Debris Removal, the NASA Orbital Debris Program Office, Quarterly News, Houston, April. 2011, 15(2011), Issue 2, pp.4-5.

4) Kimura, S., Terakura, M., and Sawada, H.: High-Performance Visual Monitoring System For IKAROS, Advances in Microelectronic Engineering, July, 2013. 1(2013), Issue 3.

5) Kimura, S., Terakura, M. and Miyasaka, M.: A High-Performance Image Acquisition And Processing System For Ikaros Fabricated Using Fpga And Free Software Technologies, 61st International Astronautical Congress (2010), Prague, CZ, IAC-10.D1.2.10.

6) Kimura, S., Terakura, M., Miyasaka, A., Sakamoto, N., Miyashita, N., Funase, R., and Sawada, H.: A high-performance image acquiring and processing unit using FPGA technologies, ISCOPS 2010, Montreal, Canada, C3.2.

7) Kasai, T., Tsujita, D., Uchiyama, T., Harada, M., Kawamoto, S., Ohkawa, Y., and Inoue, K.: Feasibility Study of Electrodynamic Tether Technology Demonstration on H-II Transfer Vehicle, 6th IAASS International Space Safety Conference (2013)

8) Ohkawa, Y., Kawamoto, S., Matsumoto, K., Shiomi, H., and Kitamura, S.: R\&D of Electrodynamic Tether for On-orbit Demonstration, Proceedings of the 5th Space Debris Workshop 2014, C3.

9) Tsujita, D., Harada, M., Kawamoto, S., and Ohkawa, Y.: The Plan of Electrodynamic Tether Experiments on HTV for Debris Removal, Proceedings of the 5th Space Debris Workshop, March, 2014, C4

10) Kawamoto, S., Ohkawa, Y., Nakanishi, H., Katayama, Y., Kamimura, H., Kitamura, S., and Kibe, S.: Active Debris Removal By A Small Satellite, 63rd International Astronautical Congress (2012), Naples, Italy, IAC-12- A6.7.8. 